\title{
August Wilhelm Ambros und Eduard Hanslick in Wien: Das Schlusskapitel einer musikästhetischen Kontroverse
}

\section{August Wilhelm Ambros and Eduard Hanslick in Vienna: The Final Chapter of a Music-Aesthetic Controversy}

Markéta Štědronská / marketa.stedronska@univie.ac.at

Department of Musicology, University of Vienna, AT

\begin{abstract}
Until now the exchange between Eduard Hanslick and August Wilhelm Ambros has been interpreted mainly in connection with their writings on musical aesthetics from the 1850s. However, sources recently during preparations for a historical-critical edition of Ambros's Vienna essays on music and music reviews indicate that the dialogue between these two music critics and aestheticians continued intensively still in the 1870s, when both were active in Vienna. The present study is focused on this final Viennese period. It turns out that the same aestheticians whose writings in the 1850s provoked the conflict between advocates of aesthetics stressing form and stressing content contributed substantially, through their later exchange in Vienna, to a softening of the controversy.
\end{abstract}

\section{Keywords}

August Wilhelm Ambros, Eduard Hanslick, Vienna, music aesthetics, music criticism 
$\mathrm{Zu}$ den Polemiken, von denen die Musikästhetik des 19. Jahrhunderts getragen wurde, gehört unabdingbar jene zwischen Eduard Hanslick (1825-1904) und August Wilhelm Ambros (1816-1876). Sie flammte Mitte der 1850er-Jahre auf, zu einem Zeitpunkt, zu dem beide Ästhetiker einander schon mehr als zehn Jahre lang gekannt haben. Ihre musikkritische und -ästhetische Karriere hatte bereits in den 1840er-Jahren in Prag angefangen. Das Musikleben der böhmischen Metropole erfuhr damals einen Aufschwung dank der Begegnung mit neuromantischer Musik und Ästhetik. Als besonders befruchtend erwies sich vor allem der Einfluss der Musikästhetik Robert Schumanns. Nach dem Vorbild von Schumanns halbfiktivem Davidsbund wurde der Prager Davidsbund gegründet - ein real existierender Künstler- und Intellektuellenkreis, dem sowohl Ambros als auch Hanslick angehörten. ${ }^{1}$ Mit ihren poetischen Kritiken im Schumann'schen Stil halfen sie dabei, das davidsbündlerische Credo zu verwirklichen: „eine neue poetische Zeit vorzubereiten, beschleunigen zu helfen“2. Die Stunde dafür schien besonders günstig zu sein: 1846 besuchte Hector Berlioz Prag, wo er u. a. durch die Aufführungen der Symphonie fantastique große Erfolge erlangte. Die Prager Davidsbündler, namentlich Ambros und Hanslick, haben zu der begeisterten Aufnahme von Berlioz' Programmmusik in Prag wesentlich beigetragen. ${ }^{3}$ Acht Jahre nach Berlioz' Prager-Triumphen sah die Situation jedoch schon wesentlich anders aus. Der mittlerweile in Wien tätige Hanslick veröffentlichte sein Traktat Vom Musikalisch-Schönen ${ }^{4}$, in dem er mit der „verrotteten Gefühlsästhetik “5 abrechnete, um eine neue Ästhetik des „Musikalisch-Schönen“ zu entwerfen. Ambros war einer der ersten, der sich dazu entschloss, auf Hanslicks „revidierte Ästhetik" mit einer Gegenschrift zu reagieren. Sie erschien 1855 unter dem Titel Die Gränzen der Musik und Poesie ${ }^{6}$. Ambros plädiert darin - hauptsächlich unter Bezugnahme auf Beethovens Werke der mittleren und der späten Periode ${ }^{7}$ - für eine idealistische Musikdeutung und lehnt Hanslicks Ansatz als materialistische Irrlehre ab. ${ }^{8}$ Vor

1 Ambros, alias der Davidsbündler „Flamin“, war Kopf des Prager Davidsbundes. Zu den anderen Kernmitgliedern, ihren Werken sowie zu den sozial- und geistesgeschichtlichen Voraussetzungen des Prager Davidsbundes vgl. LOMNÄS, Bonnie - LOMNÄS, Erling - STRAUSS, Dietmar. Auf der Suche nach der poetischen Zeit. Der Prager Davidsbund: Ambros, Bach, Bayer, Hampel, Hanslick, Helfert, Heller, Hock, Ulm. Zu einem vergessenen Abschnitt der Musikgeschichte des 19. Jahrhunderts, Bd. 1: Erläuterungen, Nachlaßregesten, Konzertdokumente, Bd. 2: Texte, Kompositionen. 1. Aufl. Saarbrücken: Pfau, 1999.

2 SCHUMANN, Robert. Zur Eröffnung des Jahrganges 1835. Ders. Gesammelte Schriften. Bd. 1. Martin Kreisig (Hrsg.). 5. Aufl. Leipzig: Breitkopf \& Härtel, 1914, S. 37-39, hier S. 38.

3 Vgl. die Auswahl von Ambros' und Hanslicks frühen Berlioz-Kritiken in: LOMNÄS - STRAUSS, op. cit., Bd. 2, S. 15-37, 212-218.

4 HANSLICK, Eduard. Vom Musikalisch-Schönen. Ein Beitrag zur Revision der Aesthetik der Tonkunst. 1. Aufl. Leipzig: Weigel, 1854.

5 Ibid., S. V.

6 AMbros, August Wilhelm. Die Gränzen der Musik und Poesie. Eine Studie zur Aesthetik der Tonkunst. 1. Aufl. Prag: Mercy, 1856 [vordatiert; tatsächliches Erscheinungsjahr bereits 1855].

7 Der Programmmusik stand Ambros zum damaligen Zeitpunkt schon deutlich skeptischer gegenüber.

8 Vgl. das flammende Schlusswort: „Die Läugner des Inhaltes der Musik, die Aesthetiker des absoluten Formenspieles sind auf kunstphilosophischem Gebiete die Materialisten, wie jene auf naturwissenschaftlichem. Es hängen diese geistigen Bewegungen unter einander inniger zusammen, als es auf den ersten Blick den Anschein hat, ja als ihre Träger selbst ahnen mögen. " AMBROS, op. cit., S. 186. Zu Ambros' Hanslick-Rezeption in den Gränzen der Musik und 
diesem Hintergrund verwundert es nicht, dass Die Gränzen der Musik und Poesie bereits im 19. Jahrhundert zum Inbegriff der gesamten antiformalistischen Polemik wurden. ${ }^{9}$ Der Austausch zwischen Hanslick und Ambros, der bis heute hauptsächlich vor dem Hintergrund der Kontroverse aus den 1850er-Jahren interpretiert wurde, ${ }^{10}$ durchlief jedoch mehrere Entwicklungsphasen. Der Austausch zwischen Hanslick und Ambros, der bis heute hauptsächlich vor dem Hintergrund der Kontroverse aus den 1850er-Jahren interpretiert wurde, durchlief jedoch mehrere Entwicklung phasen weitere Entwicklungsphasen gab. Im vorliegenden Beitrag möchte ich mich insbesondere auf die letzte Phase konzentrieren, d. h. auf die Jahre 1872-1876, in denen Hanslick und Ambros in Wien gewirkt haben.

Ambros wechselte Ende 1871 nach Wien, um dort die Stelle des Musik- und Kunstreferenten bei der Wiener Zeitung anzutreten. ${ }^{11}$ Bis zu seinem Tod 1876 veröffentlichte er in der Wiener Zeitung bzw. deren Beilage Wiener Abendpost rund 150 Musikfeuilletons und ca. 100 Kurzrezensionen über Musik. ${ }^{12}$ Diese Beiträge bilden zusammen mit Hanslicks Musikkritiken aus der Neuen Freien Presse die Hauptquelle für den in Wien erneut aufgenommenen Dialog zwischen den beiden Musikästhetikern. Um diesen Dialog zu rekonstruieren, wenden wir uns zunächst jenen Feuilletons zu, in denen beide Autoren ihre Werke gegenseitig rezensieren.

Hanslick griff gleich zu Beginn von Ambros' Tätigkeit in Wien zur Feder, um seinen ehemaligen Prager Kollegen der Wiener Leserschaft als brillanten Feuilletonisten vorzustellen. In seiner Besprechung ${ }^{13}$ von Ambros' gesammelten Aufsätzen über Musik und bildende Kunst, die kurz zuvor unter dem Titel Bunte Blätter ${ }^{14}$ erschienen waren, hebt Hanslick einerseits die Jean-Paul'schen Stilmerkmale (Vorliebe für Zitate, Witze, Abschweifungen etc.), andererseits die „wissenschaftliche Tüchtigkeit“ von Ambros'

Poesie vgl. ŠTĚDRONSKÁ, Markéta. August Wilhelm Ambros im musikästhetischen Diskurs um 1850 (Münchner Veröffentlichungen zur Musikgeschichte 75). 1. Aufl. München: Allitera, 2015, S. 142-167.

9 Vgl. hierzu insbesondere Otakar Hostinský: „Seine [Ambros'] Schrift über „die Grenzen der Musik und Pö̈sie" [...] bietet die von den Gegnern HANSLICK'S in's Treffen geführten Gründe zugleich in so grosser Zahl und von so verschiedenen Gesichtspuncten, dass man sie füglich als den ersten Repräsentanten der gesammten gegen das Buch ,Vom Musikalisch Schönen' gerichteten Polemik ansehen darf." HOSTINSKÝ, Otakar. Das Musikalisch-Schöne und das Gesamtkunstwerk vom Standpunkte der formalen Aesthetik. 1. Aufl. Leipzig: Breitkopf \& Härtel, 1877, S. 15.

10 Vgl. etwa ABEGG, Werner. Musikästhetik und Musikkritik bei Eduard Hanslick (Studien zur Musikgeschichte des 19. Jahrhunderts 44). 1. Aufl. Regensburg: Bosse, 1974, S. 29-35; BOISITS, Barbara. „Tönend bewegte Formen“ oder „seelischer Ausdruck“. Zu einer musikästhetischen Streitfrage im 19. Jahrhundert. De musica disserenda, 2006, Jg. 2, Nr. 2, S. 43-52.

11 Außerdem unterrichtete Ambros den Kronprinzen Rudolf in Musik- und Kunstgeschichte, lehrte Musikgeschichte am Konservatorium der Gesellschaft der Musikfreunde, hielt Vorlesungen an der Universität, komponierte, und setzte - nicht zuletzt - seine musikgeschichtliche Forschung in Wien fort.

12 August Wilhelm Ambros. Musikaufsätze und -rezensionen 1872-1876. Historisch-kritische Ausgabe. Bd. 1: 1872 und 1873. Markéta Štědronská (Hrsg.) (Wiener Veröffentlichungen zur Musikwissenschaft 45). 1. Aufl. Wien: Hollitzer, 2017. Bd. 2: 1874-1876 Druck in Vorb.

13 HANSLICK, Eduard. Feuilleton Musik. Neue Freie Presse, Jg. IX, Nr. 2649, 9. 1. 1872.

14 AMBROS, August Wilhelm. Bunte Blätter. Skizzen und Studien für Freunde der Musik und der bildenden Kunst. 1. Aufl. Leipzig: Leuckart, 1872. 
Feuilletons hervor. Ein günstigeres Empfehlungsschreiben an das Wiener Publikum hätte Ambros kaum erhalten können. Durch die Fokussierung auf Ambros' feuilletonistische Arbeit wird in Hanslicks Rezension allerdings die Kontroverse zwischen Inhaltsund Formalästhetik in den Hintergrund gedrängt. Ambros hingegen nahm nach Jahren gerade dieses heikle Thema wieder auf. Als Hanslicks Vom Musikalisch-Schönen 1874 in der vierten Auflage ${ }^{15}$ erschienen war, verfasste er darüber eine umfassende Rezension ${ }^{16}$, in der er die „fruchtbaren Gedanken“ und „geistvollen Züge“ des Buches lobt. Die Einstellung des ehemaligen Hanslick-Kritikers scheint sich vollkommen gewandelt zu haben. Ambros führt diesen Urteilswandel ausschließlich auf die vorgenommenen Textänderungen der vierten Auflage zurück. Diese sei eine „wesentlich verbesserte“, ja sie „gleiche kaum" den drei vorausgegangenen. Ambros interpretiert Hanslicks Revision als eine Entschärfung des formalistischen Standpunkts, wobei er folgende Gründe für diese Entwicklungstendenz anführt:

„Als Hanslik [sic] seine Schrift später, nicht mehr in dem Lärm und Tumult des allgemeinen Kampfes, wieder einmal vornahm, als die Programm-Symphonien außer Mode kamen und die kurzen Klavierstücke mit Ueberschriften - beiläufig gesagt: auch schon bei François Couperin und Rameau wohl bekannt - seltener wurden, als man gelegentlich wieder von bedeutenden Tonstücken in größeren Formen hörte, die einfach Symphonie, Sonate u. s. w. hießen und der Phantasie des Hörers nicht zumutheten, dazu ganze Novellen zu dichten, schliff Hanslik die schärfsten Spitzen weg.

Hätte die erste Edition geklungen wie diese vierte, ja wie ihre Vorgängerinnen, es wäre viel Papier erspart worden, das auf Gegenschriften verwendet wurde. Eine Verständigung über die angeregten Grundfragen würde, wie die Sachen jetzt stehen, auch mit denjenigen leicht, welche früher der Auffassung Hansliks als Gegner gegenüberstanden. "17

Diese Darstellungsweise und Argumentation ist in zweierlei Hinsicht problematisch: Zum einen weist Hanslick in der zweiten Auflage, in welcher er die gravierendsten Änderungen vorgenommen hat, eher auf einen wachsenden Einfluss der Programmmusik hin, ${ }^{18}$ zum anderen geht seine Revision genau in die entgegengesetzte Richtung, als Ambros es darstellt. Bekanntlich hatte sich Hanslick unter dem Einfluss des Formalisten Robert Zimmermann dazu entschlossen, manche idealistischen Passagen nachträglich zu tilgen, ${ }^{19}$ um den Standpunkt der Formalästhetik konziser zum Ausdruck zu bringen.

15 Leipzig: Barth, 1874.

16 AMBROS, August Wilhelm. Feuilleton Schriften über Musik I. Wiener Abendpost. Beilage zur Wiener Zeitung, Jg. CLXXII, Nr. 142, 24. 6. 1874.

17 Ibid.

18 „Als ich diese Abhandlung schrieb, waren die Wortführer der Zukunftsmusik eben am lautesten bei Stimme und mußten wohl Leute von meinem Glaubensbekenntniß zur Reaction reizen. Nun, wo ich die 2. Auflage zu veranstalten habe, sind zu Wagners Schriften noch Lißt's Programm-Symphonien hinzugekommen, welche vollständiger, als es bisher gelungen ist, die selbständige Bedeutung der Musik abdanken, und diese dem Hörer nur mehr als gestaltentreibendes Mittel eingeben. Man möge es mir zu Gute halten, wenn ich Angesichts solcher Zeichen keine Neigung fühlte, den polemischen Theil meiner Schrift zu kürzen oder abzuschwächen. "HANSLICK, Eduard. Vom Musikalisch-Schönen. Ein Beitrag zur Revision der Aesthetik der Tonkunst. 2. Aufl. Leipzig: Weigel, 1858, S. IX.

19 Vgl. hierzu etwa SCHÄFKE, Rudolf. Eduard Hanslick und die Musikästhetik. 1. Aufl. Leipzig: Breitkopf 
Durch die nachträglichen Änderungen in Vom Musikalisch-Schönen kann Ambros' Urteilswandel folglich kaum motiviert gewesen sein. Die eigentlichen Gründe dafür sind eher in Ambros' Relektüre und Reinterpretation der Erstfassung des Traktats zu suchen. Beim genaueren Hinsehen zeigt sich, dass sämtliche Stellen, die Ambros in der Rezension der vierten Auflage aus dem Traktat zitiert und zustimmend kommentiert, bereits in der ersten Auflage enthalten sind. Ambros bejaht insbesondere Hanslicks Aussage: „Unterdessen vermengt man unablässig Gefühlsaffection and musikalische Schönheit, anstatt sie in wissenschaftlicher Methode getrennt darzustellen. ${ }^{\text {"20 }}$ Er warnt vor einer Missinterpretation dieser Kernaussage, ohne einzuräumen, dass auch er sich 1855 in den Gränzen der Musik und Poesie zu einer sehr verengten Darstellung von Hanslicks Thesen hatte verleiten lassen. ${ }^{21}$ Aus der Rezension der vierten Auflage geht klar hervor, dass Ambros mit der Zeit völlig begriffen hat, dass Hanslick zwei verschiedene Blickwinkel beschreibt, aus denen ein Kunstwerk betrachtet werden kann, und dass die Ästhetik des Musikalisch-Schönen Resultat einer wissenschaftlichen Perspektivierung ist. Ambros charakterisiert diese wissenschaftliche Herangehensweise am Schluss seiner Rezension als eine philosophische. ${ }^{22}$

Die Tatsache, dass die zweidimensionale Anlage von Hanslicks Musikverständnis, auf die Alexander Wilfing hingewiesen hat ${ }^{23}$ bereits im 19. Jahrhundert völlig erkannt wurde, ist für den Kontext der Hanslick-Rezeption durchaus von Bedeutung. In Ambros' später („Wiener“) Hanslick-Rezeption handelt es sich sogar um einen zentralen Punkt. Dies zeigt sich nicht nur in der Rezension der vierten Auflage von Vom MusikalischSchönen, sondern auch in der Besprechung ${ }^{24}$ von Hanslicks gesammelten Opernkritiken Die moderne Oper ${ }^{25}$ aus dem Jahr 1875. Ambros veranschaulicht darin den Unterschied zwischen einer musikkritischen und einer wissenschaftlichen Herangehensweise an die Musik durch den Vergleich dieses Sammelbandes mit Vom Musikalisch-Schönen. Während das Traktat ein Buch von „Grund- und Principienfragen“ sei, in dem die Urteile „nur zur näheren Erläuterung und Bekräftigung der verfochtenen These“ dienen, könne man aus Die moderne Oper ein „vollständiges musikalisch-ästhetisches Credo“ Hanslicks herauslesen. Auch wenn Ambros nicht mit allen kritischen Urteilen von Hanslick einverstanden ist, bezeichnet er Die moderne Oper als „eine der glänzendsten, gedankenund gehaltreichsten Publicationen über Musik aus neuester Zeit“. Als Musikkritiker frage

\& Härtel, 1922, S. 27f.; HANSLICK, Eduard. Vom Musikalisch-Schönen. Ein Beitrag zur Ästhetik der Tonkunst. Dietmar Strauß (Hrsg.). Teil 1: Historisch-kritische Ausgabe. Mainz u. a.: Schott, 1990, S. $92 \mathrm{ff}$.

20 HANSLICK, Vom Musikalisch-Schönen (Anm. 4), S. 7.

21 Vgl. hierzu ŠTĚDRONSKÁ, op. cit., S. 149-154.

22 „Der Punkt, welcher dem Buche seinen hohen Werth giebt und eine wahre Geistesthat heißen darf, ist, daß Hanslik [...] zeigt, man müsse zur Erkenntniß des Wesens der Musik, wie sie nicht der stets empfindungsvolle, stets warm fühlende, aber selten kalt denkende Musiker und nicht der Genuß suchende Kunstliebhaber, wohl aber der Philosoph braucht, einen anderen Weg nehmen, als durch die beliebte Gefühls-Antichambre, und daß er den richtigen Weg mit fester Hand zeichnet." AMBROS, Schriften über Musik I. (Anm. 16).

23 WILFING, Alexander. Gefühl und Musik bei Eduard Hanslick und Arthur Schopenhauer. MusikE्F̈̈sthetik, 2013, Jg. 17, Nr. 66, S. 31-46, hier S. 39-45.

24 AMBROS, August Wilhelm. Feuilleton Eduard Hanslick. Die moderne Oper [Rezension]. Wiener Abendpost. Beilage zur Wiener Zeitung, Jg. CLXXIII, Nr. 78, 7. 4. 1875.

25 HANSLICK, Eduard. Die moderne Oper. Kritiken und Studien. 1. Aufl. Berlin: Hofmann, 1875. 
Hanslick nach „bedeutendem Charakter" und „geistiger Tiefe“. Allerdings ist Ambros weit davon entfernt, in Hanslicks Musikkritik eine „Negierung“ seiner Ästhetik zu erblicken. Ganz im Gegenteil, er deutet sie als deren logische Konsequenz:

„[... , tönend bewegte Formen' ist der Schlüssel für Hansliks [sic] ganze Gedanken- und Geschmacksrichtung, wenn man nur jene Thesis richtig versteht; denn nichts wäre irriger, als zu meinen, daß etwa wohlgefügte Fugen, tadellose Canons, Melodien, che quadrano, wie die Italiener sagen, Instrumentalsätze nach regelrechtem Schema für ihn das Erste und Letzte sind. Im Gegentheil! Er fragt vor Allem nach Geist, Schönheit, nach bedeutendem Inhalt.

Und eben um jenes Principes willen ist es vorzüglich die sinnlich schöne Erscheinung der Musik, welche ihn anzieht. ${ }^{\text {"26 }}$

Es ist festzuhalten: Ambros' Kritik an Hanslick hat sich keineswegs nur deswegen entschärft, weil in sein Blickfeld Hanslicks zuweilen gefühlsbetonte Musikbeschreibungen in den Kritiken geraten sind, sondern vor allem deswegen, weil er in Hanslicks Traktat Anhaltspunkte sowohl für einen formalistischen als auch für einen „idealistischen“ Musikzugang gefunden hat.

Mit welchem Interesse die beiden Musikästhetiker ihre musikkritischen Urteile gegenseitig verfolgt haben, lässt sich anhand von direkten Querverweisen in ihren Wiener Musikfeuilletons nachweisen. Es ist bezeichnend, dass beide Kritiker nach Bestätigung eigener Ansichten immer dann gesucht haben, wenn es galt, diverse weitverbreitete Klischees und Vorurteile der Musikkritik gegenüber zu bekämpfen. Ein Beispiel hierfür ist die Offenbach-Verhöhnung in der norddeutschen Musikkritik. Als sich Hanslick 1872 anlässlich der Wiener Erstaufführung von Offenbachs Fantasio von dieser missachtenden Einstellung entschieden distanzierte, berief er sich auf Ambros' Autorität:

„Es ist erfreulich, einen strengen und gelehrten Musikschriftsteller, wie Dr. A mbros, hier eine Ausnahme machen zu sehen. Wir unterschreiben vollständig, was er zu Gunsten von Offenbach's, entschiedenem und wahrlich nicht geringzuschätzendem, vielmehr in solcher Weise noch gar nicht dagewesenem Talent für komische Production' sagt und schließlich die Mahnung zusammenfaßt, daß man, Offenbach den Namen eines Künstlers nicht vorenthalten dürfen؛ “27

Ambros wird hier vor allem als gelehrter Musikhistoriker in der Diskussion herangezogen. Die Tatsache, dass ein Musikgelehrter wie Ambros Offenbachs Talent völlig erkannt hat, ist für Hanslick ein Grund mehr, sich für den französischen Operettenkomponisten einzusetzen.

26 AMBROS, Eduard Hanslick. Die moderne Oper (Anm. 24).

27 HANSLICK, Eduard. Feuilleton Operette und Singspiel. Neue Freie Presse, Jg. IX, Nr. 2788, 30. 5.1872. Hanslick bezieht sich hier auf Ambros' Feuilleton Wiener musikalische Revue. Wiener Zeitung, Jg. CLXX, Nr. 45, 25. 2. 1872. 
In Ambros' Musikkritiken finden wir eine analoge Bezugnahme auf das Urteil seines Kollegen Hanslick in einer Besprechung ${ }^{28}$ von Verdis Aida. In dieser Kritik bemüht sich Ambros darum, das unter den Wagnerianern verbreitete Urteilsklischee, Aida sei eine bloße „Nachahmung Wagners“, außer Kraft zu setzen. Um seine Meinung zu objektivieren, zitiert er eine Stelle aus Hanslicks Die moderne Oper:

„In dieser neuesten Schöpfung erscheint die leidenschaftliche Beredtsamkeit und dramatische Kraft, die schon Verdi's vorhergehende Opern charakterisirt, künstlerisch gebändigt, von Schlacken gereinigt, in einer Art von ästhetischer Katharsis. Nichtsdestoweniger ist es der volle, echte Verdi. Eine Nachahmung Richard Wagner's, wie sie in manchen Kritiken behauptet wird, liegt hier nicht vor. Gewiß hat Verdi, wie jeder moderne Operncomponist von Verstand, Wagner bedeutende Anregungen zu verdanken, aber in der Ä̈da steht nicht ein Tact, für welchen der Italiener dem Deutschen direct verschuldet wäre. ${ }^{\text {"29 }}$

Allerdings lassen sich auch Bezugnahmen von polemischem Charakter belegen. Grundsätzlich gab es ein Thema, welches im Wiener Dialog zwischen Ambros und Hanslick immer wieder Spannung und polemische Reaktionen hervorgerufen hat: der Vergangenheitscharakter der Musik und das daran gekoppelte Problem einer ästhetischen Bewertung der Alten Musik. Hanslick hatte bereits 1854 in seinem Traktat die ahistorische Qualität des Musikalisch-Schönen bezweifelt:

„Es gibt keine Kunst, welche so bald und so viele Formen verbraucht, wie die Musik. Modulationen, Cadenzen, Intervallenfortschreitungen. Harmoniefolgen nützen sich in 50, ja in 30 Jahren dergestalt $a b$, daß der geistvolle Componist sich deren nicht mehr bedienen kann und fortwährend zur Erfindung neuer, rein musikalischer Züge gedrängt wird. Man kann von einer Menge Compositionen, die hoch über den Alltagsstand ihrer Zeit stehen, ohne Unrichtigkeit sagen, daß sie einmal schön waren. " ${ }^{30}$

Auf diese These kam er auch in seinen Musikkritiken immer wieder zurück, vor allem im Zusammenhang mit der Opernmusik. ${ }^{31}$ Aus Hanslicks Sicht ist der „Verbrenungsproceß“32 der Musik ein „Naturproceß“33, der vom zeitgenössischen Rezipienten zur Kenntnis genommen werden muss. In der Vorrede zu Die moderne Oper postuliert er: „Dem schönen Unsterblichkeitsglauben müssen wir entsagen“34. Gerade an dieser Behauptung entzündete sich Ambros' Kritik. Trotz seiner insgesamt sehr positiven Bewertung von Hanslicks Sammelband wendet Ambros an dieser Stelle ein:

28 AMBROS, August Wilhelm. Feuilleton Aïda. Wiener Abendpost. Beilage zur Wiener Zeitung, Jg. CLXXII, Nr. 99, 1. 5. 1874.

29 HANSLICK, Die moderne Oper (Anm. 25), S. 252.

30 Ders., Vom Musikalisch-Schönen (Anm. 4), S. 86.

31 Vgl. etwa Feuilleton Hofoperntheater. Neue Freie Presse, Jg. IX, Nr. 2784, 26. 5. 1872.

32 Ibid.

33 HANSLICK, Eduard. Feuilleton Mozart's „Così fan tutte“. Neue Freie Presse, Jg. IX, Nr. 2930, 20.10 .1872$.

34 Ders., Die moderne Oper (Anm. 25), S. VII. 
„[...] ist denn alle Musik todt und abgethan, wenn sie nicht mehr in Concertprogrammen oder auf der

Opernbühne erscheint? Ich behaupte doch: der Geist lebt ewig - auch in der Musik. “"35

Im Sinne des Idealismus geht Ambros davon aus, dass jedes Kunstwerk zwar „die Signatur der Zeit" trage, daneben aber auch eine geistige Komponente aufweise, die unvergänglich sei. ${ }^{36}$ Ambros' Vorwurf, Hanslick richte seine Aufmerksamkeit lediglich auf diese „Signatur“, wird insbesondere im Rahmen des Diskurses um Glucks Opern laut. Klagt Hanslick nach einer Wiener Aufführung von Iphigenia in Aulis: „wir ertragen den weißen Marmor nicht mehr“"37, erinnert ihn Ambros daran, dass „wer vom Musiker alter Zeit etwas haben will, zu ihm kommen [muss] und nicht warten [kann], bis er ihm entgegengebracht wird“38. Ambros zufolge ist jede Musik dem „Veralten“ unterworfen, sie kann aber dennoch lebendig bleiben, wenn die Rezeption sich zu einer anspruchsvollen Leistung verwandelt. ${ }^{39}$ Dass die Alte Musik vom Hörer „mehr“ verlangt, betont der Musikhistoriker Ambros auch in seinen Wiener Aufführungskritiken. Er zögert dabei nicht, zuweilen etwas zugespitzte Vergleiche zu machen, um den elitären Charakter der Alten Musik zu unterstreichen. Im Zusammenhang mit einer Wiener Aufführung von Bachs Kantate Liebster Gott, wenn werd' ich sterben schreibt er:

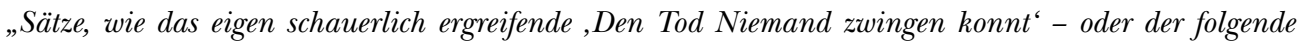
,Jesus Christus Gottes Sohn' [...] lassen Bach in seiner ganzen gigantischen Größe erscheinen. Fasse es, wer's kann, Salonmusik ist es allerdings nicht. " ${ }^{\text {"40 }}$

Diese ironische Anmerkung scheint Hanslick getroffen zu haben. Für ihn selbst war der betreffende Musikabend alles andere als ein unvergessliches Erlebnis von Bachs gigantischer Größe. Sein Missbehagen hatte allerdings eher äußere Gründe. Die Tatsache, dass Bachs Kantate zusammen mit Cherubinis Requiem erklang, fand Hanslick in dramaturgischer Hinsicht äußerst ungünstig. Seiner Meinung nach mache so viel todernste Musik das Publikum „sterbensmüde“.41 Konfrontiert mit Ambros’ oben zitierter Anmerkung wollte er sich jedoch nicht als „Salonmusikhörer“ kompromittieren lassen. Ohne Ambros direkt zu nennen, bezieht er sich auf dessen Worte in folgender Abwehrattacke:

„Mit einem ironischen ,Salonmusik ist das freilich nicht!“ widerlegt man uns am allerwenigsten. Wir wissen ganz gut, was Salon- und was Concertmusik sei, und kennen im Bereich der letzteren eine im-

35 AMBROS, Eduard Hanslick. Die moderne Oper (Anm. 24).

36 Ders., Feuilleton Opernaussichten. Wiener Zeitung, Jg. CLXX, Nr. 142, 23. 6. 1872.

37 HANSLICK, Eduard. Feuilleton Oper. Neue Freie Presse, Jg. XI, Nr. 3681, 24. 11. 1874.

38 AMBROS, Eduard Hanslick. Die moderne Oper (Anm. 24).

39 Ibid.

40 Ders., Feuilleton Musikalische Revue. Wiener Abendpost. Beilage zur Wiener Zeitung, Jg. CLXX, Nr. 72, 28. 3. 1872.

41 HANSLICK, Eduard. Feuilleton Concerte. Neue Freie Presse, Jg. X, Nr. 3099, 9. 4. 1873. 
posante Reihe classischer Tondichtungen von energisch ernstem Charakter, welche als Kunstwerke den genannten ebenbürtig sind, ohne deßhalb vom Sterben zu handeln. ${ }^{* 42}$

Die beiden gerade angeführten Zitate belegen in frappierender Weise, dass Hanslicks Alte-Musik-Abneigung, die er in seiner Autobiographie absichtlich auf die Spitze getrieben hat, ${ }^{43}$ ein Rezeptionsmodell darstellt, welches sich zum großen Teil aus dem Austausch mit dem begeisterten Musikhistoriker Ambros ergab. Die schwarz-weiße Polarisierung von Ambros' und Hanslicks Alte-Musik-Rezeption erweist sich jedoch als problematisch, zumal Hanslick auch durchaus positive Urteile über manche vorklassischen Werke fällte ${ }^{44}$ und zum Respekt vor der Tradition aufrief. Dies geht insbesondere aus seinen Korrespondenzberichten aus Frankreich und Italien hervor. Bei seinem Italien-Besuch 1874 warf er den Italienern mangelnde Pietät vor der eigenen musikalischen Vergangenheit vor. Erschrocken über die italienischen Zustände gab er den deutschen Historismus zum Vorbild:

„Und Deutschland - welche rühmliche Tätigkeit entfaltet es seit dreißig Jahren, um durch Vorträge und Aufführungen, durch kritische Ausgaben, biographische und ästhetische Werke die von unseren Vorfahren überkommenen Schätze zu sichten und fruchtbringend zu machen! Das Land jedoch, welches in manchem Sinn das Vaterland unserer Musik, im strengsten die Geburtstätte des Kunstgesanges und der Oper heißen darf, Italien, ,das Conservatorium des lieben Gottes; weiß nichts von diesem historischen Geist. "45

In Paris hat ihn umgekehrt überrascht, wie lebendig dort noch die Aufführungstradition älterer klassischer Stücke, insbesondere von Grétrys Opern, an der Opéra Comique war:

„Sie [Grétrys Opern] würden der gegenwärtigen Generation, in Wien zumal, als etwas vollständig Veraltetes erscheinen, an das wir nicht mehr anzuknüpfen wissen. Anders in Paris, wo diesen Werken nicht nur die Pietät zu Hilfe kommt (sie allein reicht niemals aus), sondern die lebendig erhaltene Tradition sowol der Sänger als des Publicums. "46

Diese Stelle ermöglicht, den Unterschied zwischen Ambros' und Hanslicks Einstellung zur Tradition besser zu verstehen. Hanslick bejaht zwar die Grétry-Pflege in Paris, er hält sie jedoch nicht für übertragbar und verpflanzbar. Während in Ambros' Augen

42 Ibid.

43 „Ich würde lieber den ganzen Heinrich Schütz verbrennen sehen, als das, deutsche Requiem; lieber Palestrinas Werke als die Mendelssohns, lieber alle Konzerte und Sonaten von Bach als Schumanns oder Brahms' Quartette [...]. Ein schreckliches Bekenntnis - nicht wahr? Wenigstens ein aufrichtiges!“ HANSLICK, Eduard. Aus meinem Leben. Peter Wapnewski (Hrsg.). Kassel u. a.: Bärenreiter, 1987, S. 405.

44 Vgl. etwa Hanslicks Bewertung von Bachs Kantate Nun ist das Heil und die Kraft im Feuilleton Concerte. Neue Freie Presse, Jg. X, Nr. 3338, 10. 12. 1873 und von Händels Cäcilienode im Feuilleton Concerte. Neue Freie Presse, Jg. XII, Nr. 4056, 10. 12. 1875.

45 HANSLICK, Eduard. Feuilleton Musikalisches aus Italien II. Neue Freie Presse, Jg. XI, Nr. 3538, 3. 7. 1874.

46 Ders.. Feuilleton Musikalische Briefe aus Paris III. Neue Freie Presse, Jg. XII, Nr. 3849, 14. 5. 1875. 
das Interesse an der Musik vergangener Epochen im Grunde in jedem Hörer latent vorhanden ist und durch Bildung und zunehmende Erfahrung kultiviert werden kann, betont Hanslick deren soziokulturelle Bedingtheit und Abhängigkeit.

Aus der Retrospektive der musikästhetischen Kontroverse in den 1850er-Jahren ist die Debatte um den Vergangenheitscharakter der Tonkunst insofern von Bedeutung, als es sich um das einzige Thema handelt, welches dem Wiener Dialog zwischen Ambros und Hanslick einen polemischen Charakter verleiht. Wenn Ambros in seiner Rezension der vierten Auflage von Vom Musikalisch-Schönen etwas moniert, dann sind es Hanslicks Ausführungen zu den Präludien und Fugen aus Bachs Wohltemperiertem Klavier. Ambros findet es nach wie vor „bedenklich“ und „unbegreiflich“, dass Hanslick sich weigere, dieser bunten Palette an Stücken verschiedene Gefühle zuzuweisen. ${ }^{47} \mathrm{Im}$ Ganzen betrachtet hat sich jedoch die Polemik über die Inhaltlichkeit der Musik dadurch entschärft, dass Ambros, wie oben bereits dargelegt, die Zweidimensionalität von Hanslicks Musikverständnis im Nachhinein erkannt hat. Demzufolge ließ er sich nicht dazu verleiten, die durchaus zahlreichen gefühlsbetonten Musikbeschreibungen in Hanslicks Wiener Musikkritiken als „Inkonsequenzen“ bzw. als Selbstnegierungen der formalen Ästhetik zu deuten. ${ }^{48}$

Die Schwierigkeit, von kritischen Urteilen auf die Entwicklung von ästhetischen Ansichten zu schließen, wird nicht nur in Bezug auf Hanslicks Musikkritik offenkundig. Auch Ambros' Wiener Musikfeuilletons belegen in vielfacher Weise, dass Ambros' ideelle Distanzierung von der Neudeutschen Schule mit seinem Urteil über bestimmte Werke Berlioz', Liszts und Wagners nicht immer kongruent war. Als Musikkritiker hütete sich Ambros davor, seine Feuilletons und Rezensionen zum Sprachrohr irgendwelcher ästhetischen Theorien zu machen. Darüber hinaus wurde ihm mit der Zeit immer bewusster, dass kritische Urteile subjektiv und wandelbar bleiben. War in Ambros' Prager Kritiken aus den 1840er-Jahren noch der selbstsichere Ton des Schumann'schen Davidsbündlertums zu vernehmen, ${ }^{49}$ wird in den Wiener Kritiken die Subjektivität und Fehlbarkeit des eigenen Urteils offen eingestanden:

„Das, was wir von der, Walküre' hörten, genügte, uns einen sehr bestimmten Maßstab für den Werth und die Wirkung des Ganzen zu geben, und so dürfen wir es uns herausnehmen, unser unmaßgebliches Urtheil über die Sache auszusprechen. "50

47 AMBROS, Schriften über Musik I. (Anm. 16). Vgl. hierzu auch ders., Gränzen der Musik und Poesie (Anm. 6), S. 60f.

48 In den Gränzen der Musik und Poesie trachtete Ambros noch danach, die „idealistischen“ Affinitäten in Hanslicks Traktat herauszuarbeiten und sie als Beweis für die innere Widersprüchlichkeit der Formalästhetik zu stellen. Ibid., S. 51.

49 Vgl. etwa Ambros' Urteil über Berlioz' Lear-Ouvertüre: „Ich habe die Partitur der Lear-Ouverture (sie liegt in diesem Augenblick vor mir) bis in die kleinsten Einzelheiten genau geprüft, die Ouverture selbst dreimal à grand orchestre ausführen hören. Ich mag mir wohl ein unbefangenes, richtiges Urtheil zutrauen. " AMBROS, August Wilhelm. Die Ouverture zu Shakespeare’s „König Lear“. Wiener Allgemeine Musikzeitung, Jg. 5, Nr. 120, 7. 10. 1845 .

50 Ders. Feuilleton Musik. Wiener Abendpost. Beilage zur Wiener Zeitung, Jg. CLXXII, Nr. 34, 12. 2. 1874. 
„Als ich später die Composition [Wagners Faust-Ouvertüre] Schwarz auf Weiß sah und unter Wagners eigener Leitung in guter Aufführung hörte, bat ich ihr das schwere Unrecht von Herzen ab, das ich ihr angethan. Ja, ich darf sagen, daß ich nun über die mächtigen Züge, welche mir hier entgegentraten, erstaunte. " ${ }^{51}$

Solche Äußerungen sollten jedoch nicht den Eindruck erwecken, dass es in der Musikkritik kein Streben nach Objektivierung gegeben habe. Wie oben angedeutet, wurden subjektive Urteile etwa durch die Berücksichtigung von Urteilen der Kritikerkollegen, oder aber durch die Einbeziehung der öffentlichen Stimme des Publikums in gewissem Sinne „objektiviert“. Dass auch der Wiener „Kritikerpapst“ Hanslick es unter dem Einfluss des Liberalismus lernen musste, auf die öffentliche Stimme des Publikums zu hören, hat Dana Gooley überzeugend dargelegt. In ihrem Aufsatz stellt sie fest: „he [Hanslick] favored the route of argument and the aspiration to impartiality"52. Den Versuch, dem „Lärm geschlossener Parteien“"53 zu entkommen, haben sowohl Ambros als auch Hanslick als eine der größten Herausforderungen der musikkritischen Arbeit wahrgenommen. Ambros hat die ideologische Polarisierung der Musikkritik wiederholt getadelt; die Absurdität des Parteienstreites thematisierte er auch in seinen Wiener Musikfeuilletons:

„,Sind Sie Wagnerianer oder nicht?' ist eine Frage, welche beantworten zu sollen man sehr oft in die Lage kommt und die, wie die Sachen stehen und was man unter einem, Wagnerianer oder Anti-Wagnerianer versteht, mit einem blanken, Ja' oder ,Nein' zu beantworten ein ehrlicher Mann, dem es um die Sache Ernst ist und der über die Sache gedacht hat, schwerlich im Stande sein wird. ,Sind Sie Wagnerianer?” das involvirt nach dem Sprachgebrauche und dem Vorstellungskreise der Fragenden die weiteren Fragen: ,Halten Sie Alles, was die Tonkunst bisher aufzuweisen hat, für schwache, zum größten Theile verfehlte Versuche, für bloße Vorstufen der endlich gefundenen wahren Musik; halten Sie Mozart für einen Zopf, Bach für einen Schulmeister u. s. w. und verehren Sie nicht blo $\beta$ - nein - beten Sie Wagner unbedingt und rückhaltlos an?' Oder: ,Sind Sie Anti-Wagnerianer?‘ - das heißt: ,Halten Sie Wagners Werke für ein Sammelsurium alles Unmelodischen, Gequälten, Verzerrten, aller Un- und Nichtmusik?' Und nun antworte der ehrliche Mann jenes blanke ,Ja“ oder ,Nein“ - wenn er kann!“54

Bei Hanslick wird das Stichwort „gute Musik“ zu einer Chiffre für die Loslösung der Musikkritik vom Parteienkampf. Um dem Vorwurf des Konservatismus vorzubeugen, betont Hanslick, dass das Epitheton „gut“ keineswegs im Sinne von „klassisch“ zu verstehen sei. ${ }^{55}$ Hanslick war sich dessen bewusst, dass eine Musikkomposition nicht a priori aufgrund ihrer Zugehörigkeit zu der konservativen oder der progressiven Musikrichtung

51 Ders. Feuilleton Musikalische Revue. Wiener Zeitung, Jg. CLXX, Nr. 291, 19. 12. 1872.

52 GOOLEY, Dana. Hanslick and the Institution of Criticism. The Journal of Musicology, 2011, Jg. 28, Nr. 3 , S. 289-324, hier S. 323.

53 HANSLICK, Aus meinem Leben (Anm. 43), S. 401.

54 AMBROS, August Wilhelm. Feuilleton Richard Wagner I. Wiener Zeitung, Jg. CLXX, Nr. 108, 12. 5. 1872. 
bewertet werden kann. ${ }^{56}$ Jede Musikkritik, die nach „guter Musik“ fragt, müsste also von dieser Polarisierung absehen. Wie konsequent Hanslick bei der Umsetzung dieses Vorhabens war, sei dahingestellt. Schon allein die Tatsache, dass eine unparteiliche Musikanschauung und -bewertung zu einem Ideal erhoben wurde, muss jedoch als ein wichtiger „Nachtrag“ zu der musikästhetischen Kontroverse der 1850er-Jahre betrachtet werden. In diesem Zusammenhang kommt also der Musikkritik eine über das subjektive Fällen von Urteilen hinausgehende Bedeutung zu. Die Frage, ob Ambros' in Wien für die Formalästhetik mehr Verständnis gezeigt hat, oder ob umgekehrt Hanslick der idealistischen Theorie gegenüber toleranter geworden ist, ist letztlich sekundär. Nicht der Kampf um die „Grund- und Principienfragen“ ${ }^{47}$ ihrer Ästhetik, sondern die Fähigkeit, sich von diesem Kampf zu befreien, wird zum Motto des in Wien geführten Dialogs zwischen Ambros und Hanslick. Insofern kann dieser Dialog eigentlich nicht mehr als „Kontroverse“ bezeichnet werden.

FWF-Projekt „August Wilhelm Ambros in Wien: Musikaufsätze und -rezensionen 1872-1876“ https://musikwissenschaft.univie.ac.at/projekte/august-wilhelm-ambros-in-wien/

\section{Bibliography}

ABEGG, Werner. Musikästhetik und Musikkritik bei Eduard Hanslick (Studien zur Musikgeschichte des 19. Jahrhunderts 44). 1. Aufl. Regensburg: Bosse, 1974.

AMBROS, August Wilhelm. Die Ouverture zu Shakespeare's „König Lear“. Wiener Allgemeine Musikzeitung, Jg. 5, Nr. 120, 7. 10. 1845.

-. Die Gränzen der Musik und Poesie. Eine Studie zur Aesthetik der Tonkunst. 1. Aufl. Prag 1856 [1855].

-. Bunte Blätter. Skizzen und Studien für Freunde der Musik und der bildenden Kunst. 1. Aufl. Leipzig: Leuckart, 1872.

-. Feuilleton Wiener musikalische Revue. Wiener Zeitung, Jg. CLXX, Nr. 45, 25. 2. 1872.

-. Feuilleton Musikalische Revue. Wiener Abendpost. Beilage zur Wiener Zeitung, Jg. CLXX, Nr. 72, 28. 3. 1872.

-. Feuilleton Richard Wagner I. Wiener Zeitung, Jg. CLXX, Nr. 108, 12. 5. 1872.

-. Feuilleton Opernaussichten. Wiener Zeitung, Jg. CLXX, Nr. 142, 23. 6. 1872.

-. Feuilleton Musikalische Revue. Wiener Zeitung, Jg. CLXX, Nr. 291, 19. 12. 1872.

-. Feuilleton Musik. Wiener Abendpost. Beilage zur Wiener Zeitung. Jg. CLXXII, Nr. 34, 12. 2. 1874.

56 Vgl. hierzu Hanslicks Begründung seiner Kritik an Liszt: „Um jeden Preis möchten wir das landläufige Mißverständniß entfernen, als entspringe die Opposition gegen Liszt's Tondichtungen aus einer engherzigen Pedanterie oder Orthodoxie, welche, angeblich aufgebracht über die Zerstörung alter Formen und Regeln, das Recht des schöpferischen Genies gegenüber der Tradition leugnet. Von alledem ist hier gar keine Rede! Stände wirklich ein Genie vor uns, wir würden um die Form, in der es erscheint, und um die Excentricitäten, die ihm anhaften, uns wenig kümmern. [...] Was wir an Liszt beanständen, ist keineswegs, dass er Großes und Schönes in ungewöhnlicher Form schaffen will, sondern daß er es nicht kann. "HANSLICK, Eduard. Feuilleton Concert und Oper. Neue Freie Presse, Jg. XIII, Nr. 4086, 11. 1.1876

57 Siehe Anm. 24. 
-. Feuilleton Aïda. Wiener Abendpost. Beilage zur Wiener Zeitung, Jg. CLXXII, Nr. 99, 1. 5. 1874.

-. Feuilleton Schriften über Musik I. Wiener Abendpost. Beilage zur Wiener Zeitung, Jg. CLXXII, Nr. 142, 24. 6. 1874.

-. Feuilleton Eduard Hanslick. Die moderne Oper [Rezension]. Wiener Abendpost. Beilage zur Wiener Zeitung, Jg. CLXXIII, Nr. 78, 7. 4. 1875.

BOISITS, Barbara. „Tönend bewegte Formen“ oder „seelischer Ausdruck“. Zu einer musikästhetischen Streitfrage im 19. Jahrhundert. De musica disserenda, 2006, Jg. 2, Nr. 2, S. 43-52.

GOOLEY, Dana. Hanslick and the Institution of Criticism. The Journal of Musicology, 2011, Jg. 28, Nr. 3, S. 289-324

HANSLICK, Eduard. Vom Musikalisch-Schönen. Ein Beitrag zur Revision der Aesthetik der Tonkunst. 1. Aufl. Leipzig: Weigel, 1854, 2. Aufl. ebd., 1858, 4. Aufl. Leipzig: Barth, 1874.

-. Feuilleton Musik. Neue Freie Presse, Jg. IX, Nr. 2649, 9. 1. 1872.

-. Feuilleton Musik. Neue Freie Presse, Jg. IX, Nr. 2666, 26. 1.1872

-. Feuilleton Hofoperntheater. Neue Freie Presse, Jg. IX, Nr. 2784, 26. 5. 1872.

-. Feuilleton Operette und Singspiel. Neue Freie Presse, Jg. IX, Nr. 2788, 30. 5. 1872.

-. Feuilleton Mozart's „Così fan tutte“. Neue Freie Presse, Jg. IX, Nr. 2930, 20. 10. 1872.

-. Feuilleton Concerte. Neue Freie Presse, Jg. X, Nr. 3099, 9. 4. 1873.

-. Feuilleton Concerte. Neue Freie Presse, Jg. X, Nr. 3338, 10. 12. 1873.

-. Feuilleton Musikalisches aus Italien II. Neue Freie Presse, Jg. XI, Nr. 3538, 3. 7. 1874.

-. Feuilleton Oper. Neue Freie Presse Jg. XI, Nr. 3681, 24. 11. 1874.

-. Feuilleton Musikalische Briefe aus Paris III. Neue Freie Presse, Jg. XII, Nr. 3849, 14. 5. 1875.

-. Feuilleton Concerte. Neue Freie Presse, Jg. XII, Nr. 4056, 10. 12.1875.

-. Die moderne Oper. Kritiken und Studien. 1. Aufl. Berlin: Hofmann, 1875.

-. Feuilleton Concert und Oper. Neue Freie Presse, Jg. XIII, Nr. 4086, 11. 1.1876

-. Aus meinem Leben. Peter Wapnewski (Hrsg.). Kassel u. a.: Bärenreiter, 1987.

HOSTINSKÝ, Otakar. Das Musikalisch-Schöne und das Gesamtkunstwerk vom Standpunkte der formalen Aesthetik. 1. Aufl. Leipzig: Breitkopf \& Härtel, 1877.

LOMNÄS, Bonnie - LOMNÄS, Erling - STRAUSS, Dietmar. Auf der Suche nach der poetischen Zeit. Der Prager Davidsbund: Ambros, Bach, Bayer, Hampel, Hanslick, Helfert, Heller, Hock, Ulm. Zu einem vergessenen Abschnitt der Musikgeschichte des 19. Jahrhunderts, Bd. 1: Erläuterungen, Nachlaßregesten, Konzertdokumente, Bd. 2: Texte, Kompositionen. 1. Aufl. Saarbrücken: Pfau, 1999.

SCHÄFKE, Rudolf. Eduard Hanslick und die Musikästhetik. 1. Aufl. Leipzig: Breitkopf \& Härtel, 1922.

SCHUMANN, Robert. Gesammelte Schriften. Martin Kreisig (Hrsg.). Bd. 1. 5. Aufl. Leipzig: Breitkopf \& Härtel, 1914.

ŠTĚDRONSKÁ, Markéta. August Wilhelm Ambros im musikästhetischen Diskurs um 1850 (Münchner Veröffentlichungen zur Musikgeschichte 75). 1. Aufl. München: Allitera, 2015.

WILFING, Alexander. Gefühl und Musik bei Eduard Hanslick und Arthur Schopenhauer. MusikË̈̈sthetik, 2013, Jg. 17, Nr. 66, S. 31-46. 
